\title{
Krisendiagnosen aus sozialpädagogischer Sicht
}

\author{
Krisen als ambivalente Erzählungen über den Zustand der Gesellschaft
}

Krisen gibt es außerordentlich viele: große und kleine; private und öffentliche; auf Gesundheit, Armut, die Gesellschaft insgesamt und auf vieles mehr bezogene. Jede_r, die/der sich dazu motiviert fühlt, kann von einer Krise sprechen.

$\mathrm{D}$ as Sprechen über Krisen erfolgt meist mit einer besonderen Intention: Der Begriffsgehalt umfasste in seinem Ursprung in der griechischen Sprache zwar auch Momente der Auswahl und der Unterscheidung. Wer aber heute eine Krise thematisiert und in der Sozialen Arbeit wird dies umfassend getan -, ruft meist zum Handeln auf, denn es scheint ein gravierendes Problem zu geben, das nicht toleriert werden dürfe. Eine Krise scheint kaum Abwägung und genaue Analyse, sondern unmittelbare Maßnahmen zu verlangen. Bewusst gegen eine solche Tendenz will der vorliegende Beitrag zum Gegenteil aufrufen: zur Vorsicht in der Sozialen Arbeit im Umgang mit der Begrifflichkeit der Krise.

\section{Ein Blick in die Geschichte}

Um dies zu begründen, mag es hilfreich sein, zunächst einen Blick in die Geschichte zu werfen, denn im Verlauf des 19. Jahrhunderts kamen drei Entwicklungen zusammen, die für das Thema der Krise aus einer sozialpädagogischen Perspektive von Bedeutung sind. So wurde erstens in der Mitte des 19. Jahrhunderts in Deutsch-

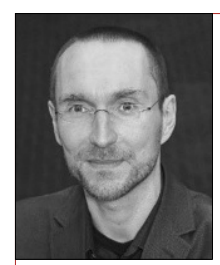

\section{Bernd Dollinger}

Universität Siegen,

Siegen, Deutschland

*1973; Dr., Professor an der Uni Siegen mit den Forschungsschwerpunkten Theorie und Geschichte der Sozialpädagogik, Jugend-/Kriminalität, Professionalisierung sowie Sozialpädagogik und Sozialpolitik.

Deutschlandbernd.dollinger@uni-siegen.de

Zusammenfassung Krisendiagnosen begleiten die Soziale Arbeit seit ihren Anfängen. Gerade derzeit wird umfassend von Krisen gesprochen. Allerdings sollte beachtet werden, welche Voraussetzungen und - möglicherweise unbeabsichtigten - Folgen dies mit sich bringt.

Schlüsselwörter Krise, Narration, Nebenfolgen land das erste Mal von einer Sozialpädagogik gesprochen, also in einer Zeit, als öffentliche Kritik an der Gesellschaft im Zuge von Industrialisierung und sozialer Frage breit vertreten wurde. Es wurde - vor dem Hintergrund eines sukzessive ausgebauten, von der Zensur bedrängten Marktes an Zeitungen und Zeitschriften (Wehler 1996, S. 540ff.) - ausgiebig darüber diskutiert, ob und ggf. auf welche Weise der Staat befugt und in der Lage wäre, die wahrgenommenen, grundlegenden Probleme zu lösen. Die Sozialpädagogik war eines der Mittel, das in Stellung gebracht wurde, um mit Hilfe von Erziehung und Unterricht sowie durch Hilfen für besonders Betroffene zumindest eine Abmilderung der scharfen sozialen Gegensätze und der breiten Armut zu erreichen.

Begleitet wurde die Institutionalisierung einschlägiger sozialpolitischer und sozialpädagogischer Mechanismen der Krisenbearbeitung, zweitens, durch eine Ausdehnung von Krisen-Zuschreibungen. Nachdem der Begriff der „Krise“ zuvor vor allem im medizinischen Bereich etabliert gewesen war, wurde er im Verlauf des 19. Jahrhunderts in seiner Bedeutung ausgeweitet und insbesondere auf soziale Zustände angewendet (Graf 2020; Koselleck 1982). Zu einer Krise wurde insbesondere die gesellschaftliche Lage, die sich, so die Diagnose, zu einem Kulminationspunkt zuspitzte, so dass eine Entscheidung drängte. In der Geschichte der Sozialen Arbeit spielt eine derartige Rhetorik eine herausragende Rolle. Mit Hilfe von Rhetoriken der Krise bringt sich die Soziale Arbeit seit Mitte des 19. Jahrhunderts immer wieder in öffentliche Diskurse ein, um besonders drängende gesellschaftliche Problemlagen anzuprangern, um zu deren Bearbeitung aufzurufen, und um nicht zuletzt sich selbst als eine solche Option der Krisenbearbeitung ins Spiel zu bringen.

Drittens aber wurde bereits frühzeitig, ebenfalls in der Mitte des 19. Jahrhunderts, auf wichtige Probleme hingewiesen, wenn man sich auf dieses (rhetorische) Kri- 
senspiel einlässt. Johann Gottfried Hoffmann, Professor in Berlin und erster Preußischer „Chefstatistiker“, warf einen nüchternen Blick auf die Krisendebatten seiner Zeit. Er konstatierte im Jahre 1845, dass nicht eine objektiv feststellbare Massen-Verarmung aufgetreten war, sondern eine Form von Armut, die bestimmte Personenkreise besonders hart traf, die aber im Wesentlichen deshalb hohe öffentliche Aufmerksamkeit fand, da sie nicht mehr wie früher toleriert werden sollte. So erscheine „uns das Elend der untern Volksklassen jetzt drückender als in frühern Zeiten, wo das Loos derselben zwar mehrentheils viel härter, aber die Neigung, es billig zu würdigen, auch viel geringer war" (Hofmann [1845] 1847, S. 226). Entscheidend für das Empfinden einer Krise war, in anderen Worten, nicht ein objektiv messbarer Problemgehalt. Wichtiger war, welche Werte man zugrunde legte, wenn man etwas problematisierte. Diese drei Entwicklungen sind nicht unabhängig voneinander zu sehen. Die Soziale Arbeit ist in Krisendebatten eingebettet, die notorisch umstritten sind und in denen festgelegt wird, ob und wie gesellschaftliche Herausforderungen angegangen werden. Wer von einer Krise spricht, nimmt meist auf moralischer Basis konkrete Lösungsvorstellungen vorweg, die gegen konkurrierende Handlungsoptionen durchgesetzt werden sollen. Ohne dass ein „tatsächliches“ Problem damit negiert werden könnte, erscheint es plausibel, deshalb genauer zu fragen, wie derartige Rhetoriken in der Sozialen Arbeit beschaffen sind.

\section{Die „Grammatik“ sozialpädagogischer Krisendiskurse}

Krisen erklären sich nicht selbst zu Krisen. So vielfältig der Begriff auch genutzt wird, man kann zumindest festhalten, dass er „über eine große zeitdiagnostische und performative Kraft verfügt“ (Bösch et al. 2020, S. 7). Krisendiagnosen beanspruchen, über den aktuellen $\mathrm{Zu}$ stand der Gesellschaft Auskunft zu geben, und indem dies kommunikativ realisiert wird, wird eine Krise anerkennungsfähig. Krisen sind in diesem Sinne „Narrationen, die sich im Laufe der Zeit verändern. Sie bilden gesellschaftlich mehrheitsfähige Erzählungen aus, die komplexe Entwicklungen auf einen Moment verdichtet reduzieren" (ebd., S. 12).

Ein Bezug auf Narrationen liegt nahe, da Krisendiagnosen zeitliche Verläufe und Dramatisierungen beinhalten und durch sie Überzeugungsarbeit geleistet werden soll - schließlich scheint es drängender gemeinsamer Anstrengungen zu bedürfen, um kollektiv gegen die angeprangerten Zustände vorzugehen. Narrationen sind "Schauplätze von Aushandlungsprozessen“ (Koschorke 2017, S. 84), und mit der Ausrufung einer Krise findet eine solche Aushandlung um den Zustand der gegenwärtigen Gesellschaft statt. Narrationen wie auch Krisendiagnosen suchen ihre Rezipient_innen aufzurütteln und sie in die jeweiligen Darstellungen einzubinden (zu Narrationen entsprechend Sukalla 2019).

Die sozialpädagogische Variante von Krisenerzählungen setzt, gleichsam mit einer besonderen „Grammatik“, in der Regel auf de-personalisierte, abstrakte Ursachen der in Frage stehenden Krise. Die Gegenwart scheint in einem schlechten Zustand zu sein, da Modernisierung, der Kapitalismus, eine beschleunigte gesellschaftliche Entwicklung oder anderes zu inakzeptablen Problemen geführt hätten. Es wird von umfassenden gesellschaftlichen Veränderungen ohne eindeutige Verantwortungsträger erzählt. Konkrete Personen kommen hingegen ins Spiel, wenn von den Auswirkungen der Krisen berichtet wird: Es gibt Modernisierungsverlierer, durch den Kapitalismus Entfremdete, durch die gesellschaftliche Beschleunigung Abgehängte usw. An ihnen kann und soll die Soziale Arbeit ansetzen.

Sie handelt sich mit dieser Erzählung zwar die Problematik ein, dass abstrakte, umfassend angelegte Problemursachen durch den Bezug auf einzelne Personen kaum realistisch gelöst werden können. Die Soziale Arbeit erscheint in dieser Hinsicht stets tendenziell überfordert zu sein, denn gegen Modernisierung, den Kapitalismus und dgl. hat sie keine ernst $\mathrm{zu}$ nehmenden Mittel. Aber sie positioniert sich gleichwohl als einen Modus der Krisenreaktion.

Krisendiagnosen verbinden demnach zeitdiagnostische Problematisierungen mit dem Aufruf zu sozialpädagogischen Interventionen. Die Gesellschaft scheint in einer fundamentalen Krise befangen zu sein, an der die Menschen leiden. Konkretisiert wird dies in sozialpädagogischen Krisendiagnosen oftmals anhand von zwei Motiven, die jeweils unterschiedliche Handlungsformen plausibel machen, selbst wenn es durchaus Überschneidungen geben kann (Dollinger 2013):

- Zum einen werden Krisen häufig so interpretiert, dass der Gesellschaft der Gegenwart ein gemeinsamer kohäsiver Kern verloren gehe. In der Geschichte der Sozialen Arbeit lag (und liegt) ein Referenzpunkt derartiger Annahmen im Fokus auf Gemeinschaften, die im Prozess gesellschaftlicher Entwicklung verloren gingen. Es scheint einen „Substanzverlust“ sozialer Bindungen zu geben, da sie immer anonymer, zweckrationaler oder abstrakter würden. Menschen würden zunehmend egoistischer handeln oder vereinsamen. In abstrakten Prozessen der Modernisierung, Individualisierung, Pluralisierung usw. erodiere, was die Gesellschaft zusammenhalte. Sofern diese Annahmen als plausibel gelten, scheint es ebenso plausibel, sozialpä- 
dagogische Gegenmaßnahmen einzuleiten, also Gemeinschaften zu restituieren, Menschen zu sozialem Engagement aufzurufen usw. Mit derartigen Postulaten wird an öffentliche und politische Diskurse angeschlossen, in denen die Soziale Arbeit sich mit ihrer Krisenrhetorik verständlich machen kann (bzw. will).

- Zum anderen werden Krisen konzipiert, die nicht von einem sozial integrationsbedürftigen Subjekt ausgehen, sondern die im Gegenteil gewissermaßen ein „Zuviel“ an Integration beinhalten. Die Gesellschaft erscheint übermächtig und bedroht die Eigensinnigkeit des Subjekts. Sie kontrolliere und/oder diszipliniere; sie benachteilige, unterwerfe und subjektiviere die Menschen, die damit - so hatte etwa Siegfried Bernfeld ([1925] 1973) argumentiert - zu Schafen werden: Sie hätten sich mehr oder weniger gleichförmig zu verhalten und dienten letztlich vorrangig dem Kapitalismus. Neuere Varianten derartiger Diagnosen erkennen ein deutlich höheres Maß an Heterogenität und individueller Vielfalt an. Aber, so wird z. B. im Anschluss an Foucault diagnostiziert, gerade hierdurch würden Menschen unterworfen, und dies als Kehrseite oder Implikat einer ihnen zugestandenen Form von (limitierter) Freiheit.

Eine klare Unterscheidung dieser beiden Arten von Krisendiagnosen ist nur idealtypisch möglich. Sie verbinden sich gleichwohl mit unterschiedlichen Begründungen, Bewertungen und Konnotationen der jeweiligen Gegenwart. Dabei ist auffällig, dass in der Sozialen Arbeit häufig mit nur mehr oder weniger stark variierten Argumenten an öffentliche Krisen- und Problemdiskurse angeknüpft wird, um sozialpädagogisches Handeln als notwendige und sinnvolle Maßnahme zu legitimieren. Charakteristisch für die Soziale Arbeit ist jeweils ein Bezug auf besondere Menschenbilder - was insofern nicht verwunderlich ist, als die Soziale Arbeit in der Regel mit einzelnen Personen(-gruppen) arbeitet und sich entsprechend durch einen expliziten Subjektbezug auszuweisen versucht (hierzu auch Schroer 2001). So ist im erstgenannten Krisentypus der „moderne“ Mensch vorrangig riskant: Er scheint außerhalb der Gesellschaft zu stehen, weshalb er bzw. sie der Re-/Integration bedürfe. Etwa Rechtsextremismus oder Gewalt werden in dieser Weise als eine Folge mangelnder Integration oder fortgeschrittener Desintegration beschrieben. Um die „Corona-Krise“ zu bekämpfen, so diese Lesart, bedarf es eines starken Zusammenhalts und gemeinsamer solidarischer Anstrengungen. Die Soziale Arbeit scheint berufen, die entsprechende Integrationsarbeit zu leisten. Im zweitgenannten Fall sind die „modernen“ Menschen hingegen gefährdet: Die gesellschaftliche Entwicklung scheint sie zu überwältigen oder zu entfremden. Sie scheinen gegen die Übermacht der Diskurse, des Kapitalismus oder z. B. der Rationalisierung keine ernsthafte Gegenwehr ausüben zu können. Die Soziale Arbeit scheint berufen, die entsprechende Aufklärungsarbeit zu leisten und notwendige „kritische“ Reaktionen zu unterstützen. Die Überwindung der Corona-Lage ist hier weniger eine Frage des gemeinschaftlichen Kampfes gegen Egoismen, sondern eher ein Problem der Kontrolle von Subjekten, die mit ihrer individuellen Freiheit abzuwägen ist.

\section{Folgerungen}

Krisendiagnosen sind ein fester Bestandteil der Art und Weise, wie die Soziale Arbeit über die jeweils gegenwärtige Gesellschaft reflektiert. Die Diagnosen begleiten sie seit ihrem Bestehen, und voraussichtlich wird dies auch weiterhin der Fall sein. Dennoch dürfte es von Bedeutung sein, an Johann Gottfried Hoffmann zu erinnern. Dass Menschen an der Gesellschaft leiden, benachteiligt und ausgegrenzt werden, ist zwar offensichtlich. Aber es sollte bedacht werden, dass es Voraussetzungen und Nebenfolgen mit sich bringt, wenn dies mit der Diagnose verbunden wird, dass die aktuelle Gesellschaft in einer Krise befangen sei - zudem mit einer Krise, die sozialpädagogischer Bearbeitung bedürfe. Zu beachten ist, dass sozialpädagogisches Handeln Nebenfolgen mit sich bringen kann und durchaus Fälle denkbar sind, in denen Herausforderungen des sozialen Lebens besser mit anderen als mit sozialpädagogischen Mitteln zu bearbeiten sind. Ob, um ein Beispiel zu nennen, Bildungsbenachteiligung tatsächlich am besten mit Hilfe Sozialer Arbeit zu begegnen ist, oder ob nicht doch strukturelle Reformen des Bildungswesens angezeigt sind, sollte gut überlegt werden. Aber selbst dort, wo sozialpädagogisches Handeln analytisch und ethisch gerechtfertigt ist, ist es keineswegs unnötig, umfassende Krisendiagnosen zu hinterfragen. So ist die sozialpädagogische Praxis stets mit der Kommunikation von negativen Kategorien verbunden. Menschen werden zu „Migranten“, „Kriminellen“, „Süchtigen“ usw. Durch derartige Kategorien können zwar Hilfe und Unterstützung mobilisiert werden. Verbunden mit der Diagnose einer umfassenden Gesellschaftskrise kann es allerdings auch nahe liegen, den so kategorisierten Menschen auf andere als freundliche Weise zu begegnen. Das Beispiel einer seit 2015 (und natürlich auch bereits zuvor) oftmals angemahnten „Migrationskrise“ mag dies illustrieren. Die Annahme einer derartigen „Krise“ mag kommuniziert werden, um den Betreffenden zu helfen und der „Krise" durch Unterstützung zu begegnen. Entscheidend ist aber, in welche konkreten Erzählungen diese Krisendiagnose eingebettet ist. Eine „Migrationskrise“ kann 
leicht als eine Bedrohungsgeschichte erzählt oder gedeutet werden, derzufolge eine Gesellschaft angeblich durch Migration gefährdet werde. Nicht die durch Migrationserfahrungen (möglicherweise) belasteten Personen werden in der Konsequenz zu Hilfebedürftigen, sondern es scheint Schutz- und Sicherheitsbedarf der Gesellschaft vor „Migranten“ zu geben. In der Rede von einer „Migrationskrise“ kann derartigen ideologischen Deutungen Vorschub geleistet werden.

Vergleichbares gilt für Krisen, bei denen gleichsam ein biologisches „Substrat“ besteht, wie in der „CoronaKrise“: Ein Virus kann vergleichsweise objektiv nachgewiesen und die von ihm ausgehenden Schädigungen können medizinisch erschlossen werden. Dennoch besteht Spielraum, wie mit diesem Wissen umzugehen ist, wer wie zu schützen ist, wie mit Infizierten umzugehen ist usw. - ein Spielraum allerdings, der nicht beliebig gefüllt werden kann. Es wird gerungen, ausgehandelt und je nach Definitionsmacht festgelegt, was richtig ist und was nicht.

Die Soziale Arbeit genießt in öffentlichen Debatten nicht immer den hohen Status und die Durchsetzungsfähigkeit, um Krisenerzählungen im Sinne ihrer Adressat_innen zu wenden. So sind Krisendiagnosen mit einer nicht abzuschüttelnden Ambivalenz versehen: Sie können zu Unterstützung aufrufen. Aber sie unternehmen dies im Rahmen der Kommunikation perspektivischer Moral- und Ordnungsvorstellungen, die sich mitunter gegen die Adressat_innen der Sozialen Arbeit wenden können. Vielleicht sollte man also trotz der Allgegenwart von Krisendiagnosen ein wenig vorsichtiger mit ihnen umgehen und Probleme direkt und spezifisch adressieren, um durch die Soziale Arbeit Hilfeleistungen zu mobilisieren.

Eingegangen. 31. März 2021

Angenommen. 27. April 2021

Funding. Open Access funding enabled and organized by Projekt DEAL.

Open Access. Dieser Artikel wird unter der Creative Commons Namensnennung 4.0 International Lizenz veröffentlicht, welche die Nutzung, Vervielfältigung, Bearbeitung, Verbreitung und Wiedergabe in jeglichem Medium und Format erlaubt, sofern Sie den/die ursprünglichen Autor(en) und die Quelle ordnungsgemäß nennen, einen Link zur Creative Commons Lizenz beifügen und angeben, ob Änderungen vorgenommen wurden.

Die in diesem Artikel enthaltenen Bilder und sonstiges Drittmaterial unterliegen ebenfalls der genannten Creative Commons Lizenz, sofern sich aus der Abbildungslegende nichts anderes ergibt. Sofern das betreffende Material nicht unter der genannten Creative Com- mons Lizenz steht und die betreffende Handlung nicht nach gesetzlichen Vorschriften erlaubt ist, ist für die oben aufgeführten Weiterverwendungen des Materials die Einwilligung des jeweiligen Rechteinhabers einzuholen.

Weitere Details zur Lizenz entnehmen Sie bitte der Lizenzinformation auf http://creativecommons.org/licenses/by/4.0/deed.de.

\section{Literatur}

Bernfeld, S. (1973). Sisyphos oder die Grenzen der Erziehung. Frankfurt a.M: Suhrkamp.

Bösch, F., Deitelhoff, N., Kroll, S., \& Thiel, T. (2020). Für eine reflexive Krisenforschung - zur Einführung. In F. Bösch, N. Deitelhoff \& S. Kroll (Hrsg.), Handbuch Krisenforschung (S. 3-16). Wiesbaden: Springer VS.

Dollinger, B. (2013). Das Politische im Disziplinären: Sozialpädagogische Identität zwischen Diffusität und eigenem „Blick“. Soziale Passagen, 5, 177-193.

Graf, R. (2020). Zwischen Handlungsmotivation und Ohnmachtserfahrung - Der Wandel des Krisenbegriffs im 20. Jahrhundert. In F. Bösch, N. Deitelhoff \& S. Kroll (Hrsg.), Handbuch Krisenforschung (S. 17-38). Wiesbaden: Springer VS.

Hoffmann, J. G. (1847). Bemerkungen über die Ursachen der entsittlichenden Dürftigkeit oder des sogenannten Pauperismus. In J. G. Hoffmann (Hrsg.), Nachlass kleinerer Schriften staatswirthschaftlichen Inhalts (S. 212-245). Berlin: De Gruyter.

Koschorke, A. (2017). Wahrheit und Erfindung. Frankfurt a.M.: S. Fischer.

Koselleck, R. (1982). Krise. In O. Brunner, W. Conze \& R. Koselleck (Hrsg.), Geschichtliche Grundbegriffe (Bd. 3, S. 617-650). Stuttgart: KlettCotta.

Schroer, M. (2001). Das Individuum der Gesellschaft. Frankfurt a.M.: Suhrkamp.

Sukalla, F. (2019). Narrative Persuasion. Baden-Baden: Nomos.

Wehler, H.-U. (1996). Deutsche Gesellschaftsgeschichte. Bd. 2. München: C.H. Beck. 